\title{
DEVELOPMENT OF LEAKY WAVE ANTENNAS FOR LAYERED RIDGE DIELECTRIC WAVEGUIDE
}

\author{
George E. Ponchak' \\ NASA Lewis Research Center, Cleveland, Ohio 44135 \\ and \\ Linda P. B. Katehi \\ University of Michigan, Ann Arbor, Michigan 48109
}

\section{INTRODUCTION}

The millimeter wave, especially above $100 \mathrm{GHz}$, and the submillimeter wave frequency spectrum offers the possibility for narrow-beam, high-resolution antennas which are critical for high definition radars required for space debris tracking, airport ground avoidance radars, and missile tracking. In addition, the frequency which most atmospheric constituents may be detected lie in this part of the frequency spectrum. Therefore, the development of electronic components for millimeter/submillimeter wave passive sensors is required for environmental monitoring of the Earth's atmosphere.

Typical microwave transmission lines such as microstrip and coplanar waveguide rely on two or more electrical conductors to concentrate and guide the electromagnetic energy. Unfortunately, the surface resistance of the conductors increases as the square root of frequency [1]. In addition, the circuit dimensions must be decreased with increasing frequency to maintain a single mode transmission line which further increases the conductor loss.

An alternative family of transmission lines are formed from two or more insulating materials and rely on the differences in the permittivities between the two materials to guide the wave. No metal conductors are required although some dielectric waveguides do utilize a metallic ground plane to facilitate the interconnections of active electrical elements or to reduce the transmission line size. Examples of such transmission lines are image guides [2], insulated image guides [3], trapped image guides [4], ridge guide [5], and layered ridge dielectric waveguide (LRDW) [6]. Although most dielectric waveguides have dimensions on the order of $\lambda$ to provide sufficient field confinement, the LRDW has been shown to provide good field confinement for electrically small lines. This offers an advantage in circuit integration.

It has been shown that a periodic array of metallic strips placed either

This work was supported by funds from the ARO (Army Research office) and NASA Lewis Research Center

This is a preprint or reprint of a paper intended for presentation at a conierence. Because changes may be made before formal publication, this is made available with the understanding that it will not be cited or reproduced without the permission of the author. 
along or on top of a dielectric waveguide forms an effective radiator [7-9]. This antenna is easy to fabricate and there is a good background of microstrip type antenna design information in the literature. This paper reports the development of the first frequency scanning antenna fed by a LRDW.

\section{FABRICATION OF LRDW}

The materials which are used for the LDRW and the dimensions of the lines are dependant on the desired frequency of operation. For the microwave region and the lower portion of the millimeter wave region, $f<100 \mathrm{GHz}$, the dimensions of the lines permit the use of teflon based soft substrates such as RT/Duroid. The advantage of these substrates is that they are low loss, inexpensive, can be purchased in a wide range of permittivities, and are easy to form into circuits. The LRDW is shown in Figure 1. The first step in the fabrication procedure is to bond a sheet of RT/Duroid 6010, $\epsilon_{\mathrm{r}}=10.8$, and a sheet of RT/Duroid 5880, $\epsilon_{r}=2.2$, together using 3001 bonding film from Rogers Corp. The bonding film is sandwiched between the two substrates which are then placed between two stainless steel plates. The assembly is then placed in an oven at $220 \mathrm{C}$ for 60 minutes. After the boards have been bonded, another sheet of bonding film is melted onto the low permittivity board. The LRDW circuit is then cut out using either a knife, milling machine, or a punch. Finally, the ridge circuit is placed onto a sheet of RT/Duroid 6010, $\epsilon_{\mathrm{r}}=10.8$, and bonded in place using the same procedure as before. It is important that light pressure be used in the last bonding operation or the previously bonded boards will slide apart when the bonding film melts.

\section{LAYERED RIDGE DIELECTRIC WAVEGUIDE ANTENNA}

A schematic of a LRDW periodically loaded array is shown in Figure 2. Although an exact field solution is required for the metal strip loaded LRDW to theoretically predict the radiation pattern for the array, the direction of the main beam may be determined easily by:

$$
\sin \left(\theta_{I}\right)=\left(\beta_{0}+2 \pi m / d\right) / k_{0} \leq 1
$$

where $\mathrm{m}$ covers the range of all integers. $\beta_{\mathrm{o}}$ is the propagation constant of the loaded transmission line although it may be approximated by the unloaded $\beta$ if the line is lightly loaded [10]. This should be the case for the LRDW since the fields are primarily confined in the low permittivity guiding layer [6].

Figure 3 shows the direction of the main beam as a function of frequency for the LRDW antenna shown in Figure 2 . The propagation constant, $\beta / k_{0}$, was determined using the EDC method [3]. Also shown in Figure 3 are three experimental points. The experimental points are in good agreement with the theory which validates the use of the unloaded $\beta$ in Equation 1. The radiation 
pattern for this antenna at $29 \mathrm{GHz}$ is shown in Figure 4. The main beam is symmetric and the side lobes are at $-16 \mathrm{~dB}$ which is in good agreement with dielectric waveguide fed strip arrays [7,8]. A measure of the efficiency of the antenna is the ratio of the insertion loss of the loaded LRDW to the unloaded LRDW. For this antenna, the excess insertion loss is $25 \mathrm{~dB}$. The excess insertion loss is a function of the number of radiators and the antenna may therefore be made more efficient by increasing the number of strips.

\section{CONCLUSIONS}

A frequency scanning antenna has been fabricated and tested at Ka-Band using a layered ridge dielectric waveguide feed to an array of radiating strips. The antenna is easy to fabricate from low cost microwave teflon substrates.

\section{REFERENCES}

[1] R. E. Collin, Foundations for Microwave Engineering, McGraw-Hill Book Co., New York, 1966.

[2] M. Dydyk, "Image guide: a promising medium for EHF circuits," Microwaves, April, 1981, pp. 71-80.

[3] W. V. McLevige, T. Itoh, and R. Mittra, "New waveguide structures for millimeter-wave and optical integrated circuits, "IEEE Trans. Microwave Theory Tech., Oct., 1975, pp. 788-794.

[4] T. Itoh and B. Adelseck,"Trapped image guide for millimeter-wave circuits, "IEEE Trans. Microwave Theory Tech, Dec., 1980, pp. 14331436.

[5] T. Wang and S. E. Schwarz, "Design of dielectric ridge waveguides for millimeter-wave integrated circuits," IEEE Trans. Microwave Theory Tech., Feb., 1983, pp. 128-134.

[6] A. G. Engel, Jr. and L. P. B. Katehi, "Low-loss monolithic transmission lines for submillimeter and terahertz frequency applications," IEEE Trans. Microwave Theory Tech., Nov., 1991, pp. 1847-1854.

[7] M. T. Birand and N. Williams, "Printed dipole millimeter-wave array using an insular guide feeder," IEE Proc., Vol. 131, Pt. H, No. 2, April 1984, pp. 107-113.

[8] A. Henderson, E. England, and J. R. James, "New low-loss millimetrewave hybrid microstrip antenna array," 11 th European Microwave Conf. Digest, Sept. 7-10, 1981, pp. 825-830.

[9] T. Rozzi, A. Morini, and G. Gerini, "Analysis and applications of 'microstrip-loaded inset dielectric waveguide'," IEEE Trans. Microwave Theory Tech., Feb., 1992, pp. 272-278.

[10] A. Hessel, "General characteristics of travelling-wave antennas," in R. E. Collin and F. J. Zucker (Eds.) Antenna Theory (part 2), McGraw-Hill, New York, 1969. 


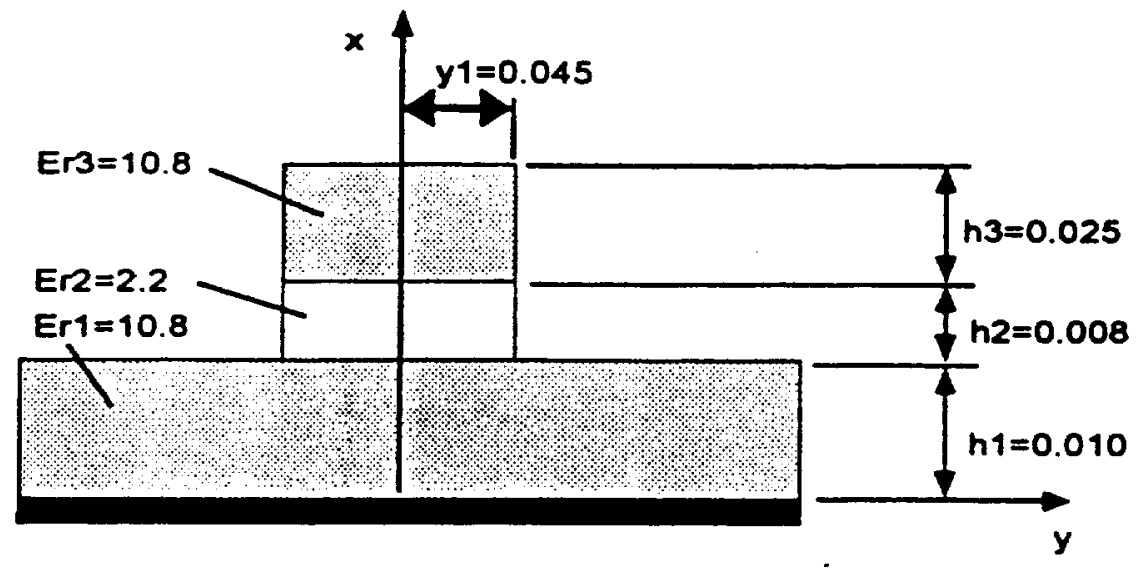

Figure 1: Layered ridge dielectric waveguide

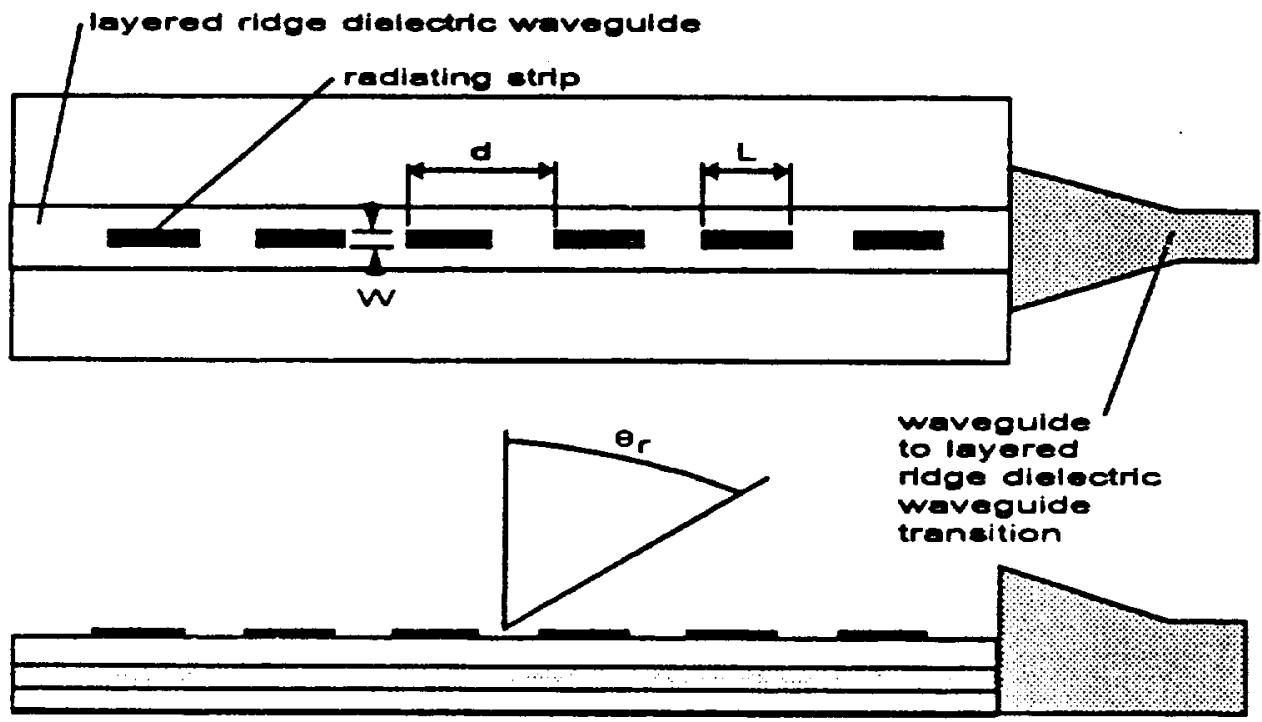

Figure 2: Layered ridge dielectric wavegulde antenna comprised of an array of conducting etrips on top of the ridoe ( $=i x$ trips, $d=0.588 \mathrm{~cm}, L=0.106 \mathrm{~cm}, W=0.068 \mathrm{~cm}$ )

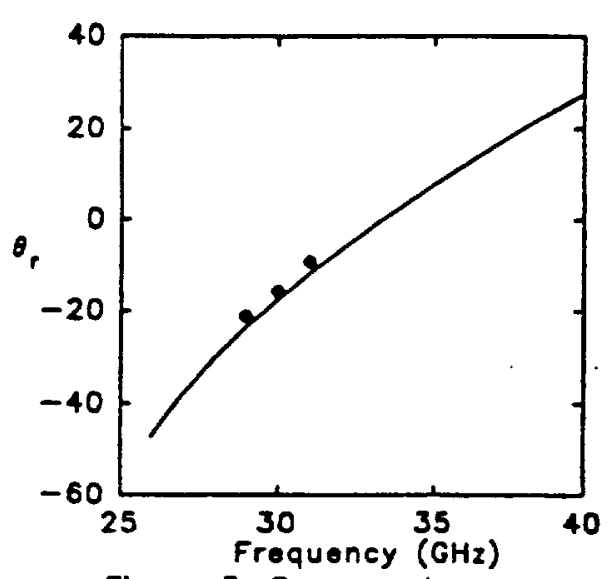

Figure 3: Scan angle varaus frequency

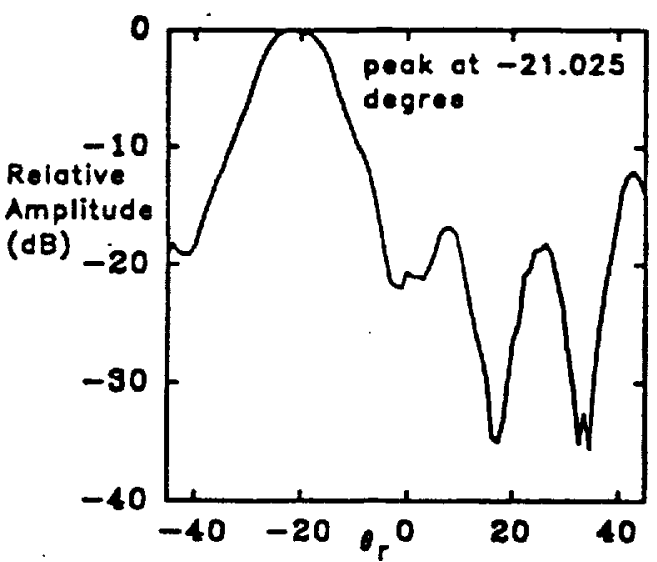

Figure 4: Rodiotion pattern for LROW ot $20 \mathrm{GHz}$ 